Molecular identification of chlamydial cause of abortion in small ruminants in Jordan

Huthaifa Salah Ababneh, Mustafa Mohammed Kheir Ababneh, Wael Mahmoud Hananeh, Fawzi Mohammad Alsheyab, Khaleel Ibraheem Jaw

Tropical Animal Health and Production

ISSN 0049-4747

Trop Anim Health Prod

DOI 10.1007/s11250-014-0654-x
Volume 46 - Number 6 - August 2014

ONLINE FIRST

\section{Tropical} Animal Health and Production et 
Your article is protected by copyright and all rights are held exclusively by Springer Science +Business Media Dordrecht. This e-offprint is for personal use only and shall not be selfarchived in electronic repositories. If you wish to self-archive your article, please use the accepted manuscript version for posting on your own website. You may further deposit the accepted manuscript version in any repository, provided it is only made publicly available 12 months after official publication or later and provided acknowledgement is given to the original source of publication and a link is inserted to the published article on Springer's website. The link must be accompanied by the following text: "The final publication is available at link.springer.com". 


\title{
Molecular identification of chlamydial cause of abortion in small ruminants in Jordan
}

\author{
Huthaifa Salah Ababneh • Mustafa Mohammed Kheir Ababneh • \\ Wael Mahmoud Hananeh • Fawzi Mohammad Alsheyab • Khaleel Ibraheem Jawasreh • \\ Moath Ahmad Al-Gharaibeh • Mohammed Mahmoud Ababneh
}

Received: 04 September 2013 / Accepted: 29 July 2014

(C) Springer Science+Business Media Dordrecht 2014

\begin{abstract}
Chlamydophila abortus (Ch. abortus) is the etiological agent of ovine enzootic abortion (OEA) and one of the most common infectious agents of abortion in small ruminants worldwide. RFLP-PCR analysis of the outer membrane protein gene (OMP2 gene) was used for diagnosis and characterization of chlamydial causes of abortion in small ruminants in Jordan. Sixty-six placental tissues and 15 vaginal swabs were collected from aborted ewes and does to identify cause of abortion in Jordan. Thirty-eight placental samples (58 \%) and 13 vaginal swabs $(87 \%)$ were positive for chlamydial DNA. Shedding of bacteria in vaginal swabs was detected within 7 days after abortion. The results of this study showed that chlamydiosis is one of the important causes of abortion in small ruminants in Jordan. In addition, vaginal swab is an excellent sample for molecular diagnosis of chlamydiosis. DNA sequencing and RFLP analysis of the $O M P 2$ reveal that all chlamydial cause of abortion in small ruminants in Jordan are due to Ch. abortus. While, Ch. pecorum was not detected in any sample. $O M P 2$ gene of the isolated Jordanian strain was identical (100\%) to Ch. abortus FAS strain. In conclusion, Ch. abortus is an important cause of abortion in Jordan; vaginal swab within 7 days of abortion can be used for molecular diagnosis of chlamydiosis in small ruminants.
\end{abstract}

H. S. Ababneh $(\bowtie) \cdot$ F. M. Alsheyab • M. A. Al-Gharaibeh National Center for Agricultural Research and Extension, Baqa' 19381, Jordan

e-mail: hothaifaababneh@yahoo.com

M. M. K. Ababneh - W. M. Hananeh • M. M. Ababneh Faculty of Veterinary Medicine, Jordan University of Science and Technology, Irbid, Jordan

K. I. Jawasreh

Faculty of Agriculture, Jordan University of Science and

Technology, Irbid, Jordan
Keywords Chlamydophila abortus · Ovine enzootic abortion · Outer membrane protein gene $\cdot$ PCR-RFLP · DNA sequencing $\cdot$ Jordan

\section{Introduction}

Chlamydophila is an obligate, intracellular gram-negative bacterium that causes a broad range of diseases in both human and animals. RFLP-PCR test based on 16S-23S ribosomal intergenic region was used to identify Chlamydiaceae species; according to this phylogenic analysis, the family Chlamydiaceae consists of two genera: Chlamydia $(C$.) and Chlamydophila (Ch.), and nine species (Longbottom et al. 2002). The most economically important chlamydial agent in small ruminants is Chlamydophila abortus (Ch. abortus; previously classified as Chlamydia psittaci immunotype 1) (Aitken and Longbottom 2007; Stuen and Longbottom 2011). Ch. abortus is the etiological agent of ovine enzootic abortion (OEA) and one of the most common infectious agents of abortion in small ruminants worldwide. In sheep, the disease is usually manifested as abortion in the last 2 to 3 weeks of gestation. While goats can abort at any stage of pregnancy, most abortions occur during the last 2 to 3 weeks of gestation (Matthews 1999; Nietfeld 2001). Another member of the genus Chlamydophila is Chlamydophila pecorum. Ch. pecorum is commonly isolated from the digestive tract of clinically inconspicuous ruminants (Mohamad and Rodolakis 2010). Although Ch. pecorum had been isolated from placenta and vaginal fluid of aborted small ruminants, involvement of Ch. pecorum in small ruminant's abortion is still unclear (Berri et al. 2009). In addition, Ch. pecorum has been implicated as a cause for infertility, conjunctivitis, arthritis, mastitis, and pulmonary inflammation in sheep, goats, and cattle (Berri et al. 2009). Human infection of Ch. abortus can be acquired when dealing with contaminated sources such as 
placentas and fetuses. The symptoms in human range from influenza-like symptoms to life-threatening illness and abortion in pregnant women (Pospischil et al. 2002; Stone et al. 2012). Vaccination is the best option in controlling OEA (O.I.E 1996). Currently, two types of chlamydial vaccines are commercially available; inactivated and live attenuated. Both vaccines have been documented to prevent abortion and to reduce transmission (O.I.E 1996; Longbottom and Livingstone 2006).

Direct examination, bacterial isolation, serological and molecular tests have been described for diagnosis of chlamydiosis. Serological testing is the most commonly used technique for diagnosis and epidemiological studies. In fact, complement fixation test is recommended by the World Organization for Animal Health (OIE). However, vaccinations against $C h$. abortus may interfere with serological investigations. Molecular techniques such as conventional and realtime PCR have been used to identify Ch. abortus in abortion cases. Amplification of the outer membrane protein 2 gene $(O M P 2)$ was found to be a reliable procedure to diagnose Ch. abortus infection. Furthermore, enzymatic digestion of the PCR product can further differentiate Ch. abortus from Ch. pecorum (Hartley et al. 2001; Marsilio et al. 2005).

Majority of abortion cases in small ruminants are reported in the last month of pregnancy. Infectious diseases such as brucellosis, toxoplasmosis, and chlamydiosis are the usual suspects in Jordan. These diseases can be of considerable economic importance to the sheep and goat industries as well as public health due to their zoonotic nature (Aldomy et al. 2009). Chlamydiosis in small ruminants in Jordan has been documented serologically (Al-Qudah et al. 2004). But, their contribution in abortion has not been investigated. Therefore, the aims of this study were to identify chlamydial cause of abortion in small ruminants in Jordan using RFLP-PCR analysis and DNA sequencing of the $O M P 2$ gene.

\section{Materials and methods}

Animals

Flocks of sheep $(n=17)$ and goats $(n=5)$ with recent cases of abortion in the northern parts of Jordan were used in the current investigation.

Placental and vaginal sample collection

A total of 66 placental samples (cotyledons and intercotyledonary areas) from aborted ewes $(n=46)$ and does $(n=20)$ were collected within $24 \mathrm{~h}$ of abortion in plain tube and stored at $-80^{\circ} \mathrm{C}$ until DNA extraction and PCR analysis. In addition, vaginal swabs were collected from aborted ewes ( $n=9$ from three flocks) and does ( $n=6$ from two flocks) within 7 days after abortion. Swabs were placed in transport medium (Vircell microbiologists, Spain) and kept on ice. Transport medium containing swabs were centrifuged at $10,000 \mathrm{rpm}$ at $4{ }^{\circ} \mathrm{C}$ for $3 \mathrm{~min}$. Thereafter, pellets were stored at $-80^{\circ} \mathrm{C}$ until used for DNA extraction and PCR analysis.

\section{DNA isolation}

Total DNA was isolated from placenta and vaginal pellet using DNeasy Blood and Tissue Kit (Qiagen Inc., Mississauga, Canada) according to the manufacturer's instructions. Genomic DNA was visualized in $1 \%$ agarose gel to ensure its quantity and quality. Extracted DNA was stored at $-20^{\circ} \mathrm{C}$ until PCR analysis.

\section{PCR analysis}

Detection of chlamydial DNA was performed by amplification of the $O M P 2$ with specific forward and reverse primer, F: 5'ATG TCC AAA CTC ATC AGA GGA G-3' R: 5'-CCT TCT TTA AGA GGT TTT ACC CA- ${ }^{\prime}$. The PCR was performed in a total reaction volume of $20 \mu \mathrm{l}$ containing $3 \mu \mathrm{l}$ of template, $4 \mu \mathrm{l}$ of $5 \times$ Firepol master mix (Solis Biodyne, Tartu, Estonia) and 10 pmol of each primer. Thermal cycling conditions were initial denaturation $94{ }^{\circ} \mathrm{C}$ for $2 \mathrm{~min}$; followed by 40 cycles with 1 cycle consisting of $1 \mathrm{~min}$ at $94{ }^{\circ} \mathrm{C}$ for denaturation, $1 \mathrm{~min}$ at $56^{\circ} \mathrm{C}$ for primer annealing and $1 \mathrm{~min}$ at $72{ }^{\circ} \mathrm{C}$ for extension. The last cycle included 5-min incubation at $72{ }^{\circ} \mathrm{C}$ for final extension. Positive control of Chlamydophila DNA (Vircell microbiologists, Spain) and negative control of nontemplate control was performed with each PCR run. The amplified DNA product was analyzed by agarose gel electrophoresis, and the product was visualized under UV light.

\section{Purification of amplified DNA}

Amplified DNA was purified from agarose gel using PCR purification kit (Jena Bioscience GmbH, Germany) according to the manufacturer's instructions. Then, the purified product was stored at $-20^{\circ} \mathrm{C}$ until DNA sequencing and RFLP analysis.

DNA sequencing

The purified PCR amplicons were sent for sequencing (Macrogen, Korea) to confirm the PCR results. Sequences were viewed with BioEdit software and aligned using the MegAlign software of the DNASTAR package. Nucleotide identity and phylogenetic analysis were done with the abovementioned software.

\section{RFLP-PCR analysis}

PCR-RFLP was used to differentiate $C h$. abortus from Ch. pecorum using the amplified OMP2 gene. This method is able to differentiate Ch. abortus from Ch. pecorum by 


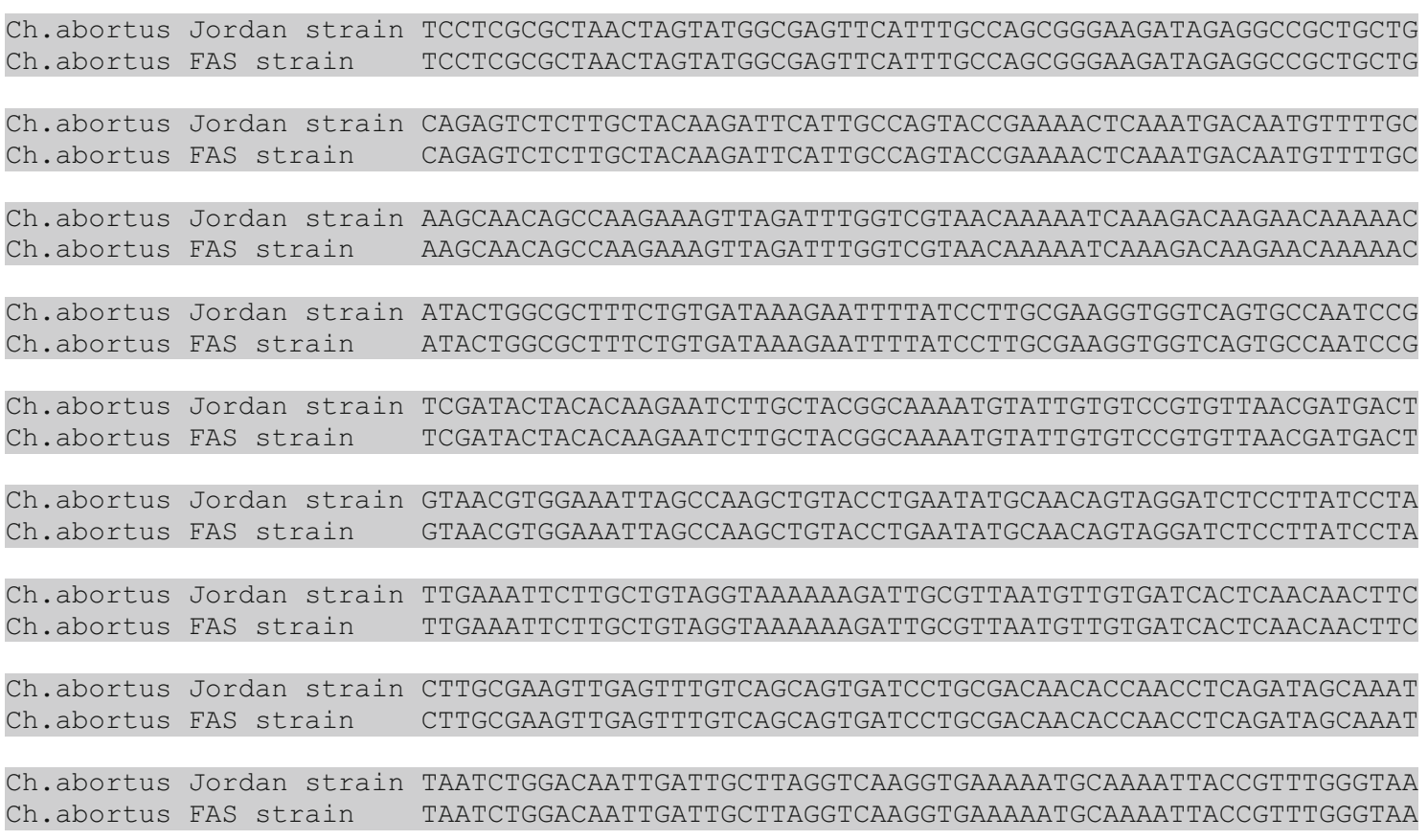

Fig. 1 The alignment of the partial sequence (540 nt) of the $O M P 2$ gene of the Ch. abortus Jordan strain (GenBank number KJ739313) and the reference Ch. abortus FAS strain (GenBank number DQ494809 ). A

producing different band patterns using Alu1 restriction enzyme. Alu1 restriction enzyme was used as described by Marsilio et al. (2005). The digested products were visualized on $2 \%$ agarose gel electrophoresis. Ch. abortus is expected to yield two bands of 352 and 235 bp while Ch. pecorum is expected to yield two bands of 397 and $193 \mathrm{bp}$.

\section{Results}

Detection of OMP2 gene of Chlamydophila in placenta and vaginal swab

Thirty-eight placental samples $(58 \%$; ewes $=28$, does $=10)$ out of 66 and 15 vaginal swab ( $87 \%$; ewes $=7$, does $=6)$ out of 15 were positive for chlamydial DNA with a PCR product complete matching is seen in all nucleotide that gives $100 \%$ nucleotide identity between the Jordan strain and the FAS reference strain of Ch. Abortus

size of $587 \mathrm{bp}$ of the $O M P 2$ gene. Shedding of bacteria in vaginal swabs was detected within 7 days after abortion. No positive results in the OMP2 PCR were found after 7 days (data not shown). Placenta and vaginal samples were collected from the same animal in five cases. Of those, placenta and vaginal swab of two animals and vaginal swab but not the placenta in one animal were positive for chlamydiosis. Both the vaginal and placental samples of the last two animals were negative for chlamydiosis.

Sequence of $O M P 2$ gene of Ch. abortus Jordan strain

The PCR amplicon of the partial OMP2 gene was directly sequenced. A fragment with a size of $540 \mathrm{nt}$ was obtained and aligned with the same fragment of the reference Ch. abortus FAS strain (Fig. 1). MegAlign software of the DNA star

Table 1 The nucleotide identity percentages of partial OMP2 gene of the Ch. abortus Jordanian strains (KJ 739313) and other Ch. related strains

\begin{tabular}{|c|c|c|c|c|c|c|}
\hline Ch. strains & Ch. abortus Jordan strain & Ch. abortus strain FAS & Ch. felis & Ch. caviae & Ch. pneumoniae & Ch. pecorum \\
\hline Ch. abortus Jordan strain (KJ739313) & 100 & - & - & - & - & - \\
\hline Ch. abortus strain FAS (DQ494809.1) & 100 & 100 & - & - & - & - \\
\hline Ch. felis (AY286314.1) & 90.2 & 90.6 & 100 & - & - & - \\
\hline Ch. caviae (AE015925.1) & 90.2 & 90.7 & 90 & 100 & - & - \\
\hline Ch. pneumonia (U56925.1) & 75 & 75.7 & 71.7 & 76.4 & 100 & - \\
\hline Ch. pecorum (U56924.1) & 80 & 80.4 & 75.3 & 75 & 72.1 & 100 \\
\hline
\end{tabular}

The highest nucleotide identified (100\%) is seen between the Ch. abortus Jordan strain and the FAS strain while the lowest (75\%) is seen between the Ch. abortus Jordan strain and the Ch. pneumoniae 
Fig. 2 Phylogenetic tree of the partial OMP2 gene of the Chlamydophila abortus Jordanian strain and other related strains. The Ch. abortus Jordan strain lies within the same cluster as the Ch. abortus FAS strain

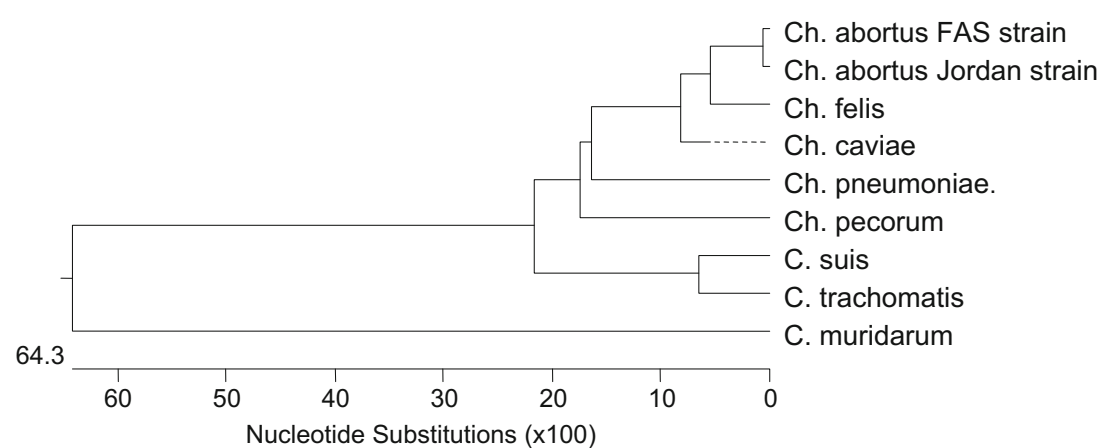

package was used to design the phylogenitic tree. The nucleotide identity percentages of partial OMP2 gene of the Jordanian strains and other related $C h$. species are shown in Table 1. OMP2 gene of the isolated Jordanian strain (GenBank number KJ739313) was identical $(100 \%)$ and grouped in the same cluster with Ch. abortus FAS strain (GenBank number DQ494809) as shown in Figs. 1 and 2 and Table 1, while the lowest identity (75\%) was between the Ch. abortus Jordan strain and the Ch. pneumoniae.

Digestion pattern of the PCR amplicon of the OMP2 gene

PCR-RFLP assay was used to differentiate between Ch. abortus and Ch. pecorum. Digestion of the PCR amplicon of the $587 \mathrm{bp} O M P 2$ gene with Alu1 yielded two bands of 235 and $352 \mathrm{bp}$ as presented in $2 \%$ agarose gel electrophoresis image (Fig. 3). The restriction digestion pattern found in all OMP2 PCR positive (both placental and vaginal) samples indicated that all these samples are infected with Ch. abortus and not Ch. pecorum.

\section{Discussion}

Diagnosis of chlamydial abortion in small ruminants depends on the detection of bacteria in impression smears of the

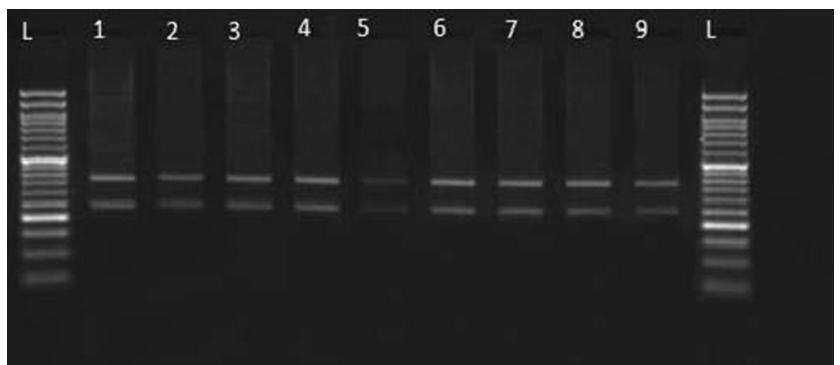

Fig. 3 RFLP-agarose gel electrophoresis image, 1-9: Alu1 digested $O M P 2$ gene yielding two bands with 235 and $352 \mathrm{bp}$. This restriction pattern is compatible with Chlamydophila abortus. L, 50 bp DNA marker. The size of the intense bands in the $50 \mathrm{bp}$ DNA ladder are 200 and $500 \mathrm{bp}$, respectively placenta, serological analysis, isolation of Chlamydophila or its nucleic acid from aborted animals or vaginal secretions. The result of this study showed that the placenta and vaginal swab within 7 days of abortion were equally suitable samples for PCR detection of Ch. abortus in small ruminant. PCR is considered to be the most sensitive diagnostic method for diagnosis of Chlamydiaceae in human medicine. Ch. abortus can be isolated from the aborting sheep and goat before treatment with antibiotics; however, culture of the pathogen is rarely performed because of technical difficulty and safety. Chlamydiosis is a zoonotic disease, and the causative agent can impair human health during the isolation and identification of Ch. abortus. Therefore, molecular assays such as PCR-RFLP offer a safer, fast, and accurate method for detection of chlamydial DNA in cases of abortion. OMP2 gene shows a high degree of conservation between Chlamydiaceae species for most of its sequence. Therefore, amplification of $O M P 2$ gene alone does not allow the discrimination between chlamydial species. However, analysis of PCR products by RFLP and digestion with Alul is sufficient for species identification. The use of PCR-RFLP in the current study identified the isolates as Ch. abortus. Only two bands were generated in PCR-RFLP with Alu1 digestion. The limited number of fragments expected with the use of Alu1 digestion allow easy discrimination among different chlamydial species (for example, Ch. abortus vs Ch. pecorum). Several investigators reported that high sensitivity and specificity of RFLP using the Alu1 enzyme (Hartley et al. 2001; Marsilio et al. 2005). Moreover, shedding of Ch. abortus through vagina was detected by PCR until 7 days after abortion. Therefore, when the aborted fetus and placenta are lost, vaginal swab is considered to be the optimal sample for diagnosis of chlamydial abortion. Vaginal chlamydial shedding decreases rapidly after abortion, and few chlamydial DNA were detected in the vaginal swabs at the subsequent estrus after abortion (Livingstone et al. 2009). In one animal, Ch. abortus was detected in the vaginal swab but not in the placenta. In accordance with the current study, Marsilio et al. (2005) showed that the high collagen content in the placenta could impair DNA quality making vaginal swab a better sample than placenta for PCR (Marsilio et al. 2005). 
The most important studied causes of infectious abortion in small ruminant in Jordan are Brucellosis (Samadi et al. 2010) and Toxoplasmosis (Abu-Dalbouh et al. 2012). The cause of abortions in the current study was not fully investigated. However, the results of this study showed, for the first time, that Ch. abortus is considered to be a major cause of abortion in small ruminants in Jordan. This is supported by the result of an old published serological survey in Jordan (Al-Qudah et al. 2004). In the latter study, all the tested flocks were found to be infected with $C h$. abortus and the prevalence ranged from 4 to $57 \%$ and 4 to $20 \%$ in sheep and goat flocks, respectively (AlQudah et al. 2004). In addition, more than $60 \%$ infection rate was determined serologically in sheep and goat flocks used in the current study (unpublished data). Lack of control strategy of chlamydiosis through vaccination may be the cause behind the high infection rate of chlamydiosis especially in areas where brucellosis is controlled leaving Ch. abortus as the major infectious cause of abortion in Jordan.

PCR-RFLP analysis reveals that all chlamydial cause of abortion in Jordan was due to Ch. abortus. Restriction with Alu1 gives species-specific band lengths. RFLP yielded two bands of 352 and 235 bp which is consistent with Ch. abortus. On the other hand, RFLP of Ch. pecorum is expected to yield two bands of 397 and 193 bp. Berri et al. (2009) in Tunisia identified Ch. pecorum from the placenta of aborted small ruminants. Rodolakis and Souriau (1989) showed that intradermal or intravenous inoculation of pregnant ewes with Ch. pecorum strains isolated from ovine abortion cases did not induce the same abortion rate as Ch. abortus (Rodolakis and Souriau 1989). However, the role of Ch. pecorum in small ruminants abortion is still unclear; therefore, further studies are required.

The results of this study and other related studies showed that $O M P 2$ gene is considered as conserved gene (Vladimir and Andrey 2005). Sequencing of the $O M P 2$ gene in the Jordanian strain was similar with $100 \%$ homology to the reference strain. This is consistent with the findings of Vretou et al. (1996) who reported identical pattern after Alu1 digestion of $O M P 2$ gene from four different diverse Ch. abortus strains. In addition, complete genome sequence of Ch. abortus showed high level of conservation in gene sequence in comparison to other members of Chlamydiaceae (Thomson et al. 2005). OMP2 is responsible for immunization of Chlamydia infection (Vladimir and Andrey 2005). Therefore, global Ch. abortus vaccine targeting this protein may protect ewes and does against chlamydiosis.

In conclusion, this study showed that Ch. abortus plays a substantial role in sheep and goat abortion in Jordan. OMP2 gene in the Jordanian strain is similar with $100 \%$ homology to the reference strain. Authorities in the ministry of agriculture in Jordan are required to set up appropriate vaccination and epidemiological surveillance to control and eradicate this disease.
Acknowledgments A great acknowledgement is for the Scientific Support Research Fund for financing this project (grant number Z.B/1/10/ 2010). Also, special thanks for Eng. Faiq Hijazeen, Dr. ebtehal alKhraisha for their assistance in sample collection and Dr. Amani ALDawood for her constructive critique of the manuscript.

Conflict of interest The authors declare that they have no conflict of interest.

\section{References}

Abu-Dalbouh, M.A., Ababneh, M.M., Giadinis, N.D. and Lafi, S., 2012. Ovine and caprine toxoplasmosis (Toxoplasma gondii) in aborted animals in Jordanian goat and sheep flocks. Tropical Animal Health and Production, 44, 49-54.

Aitken, I., Longbottom, D., 2007. Chlamydial abortion - diseases of sheep, Blackwell publishing USA, pp. 105-112.

Aldomy, F., Hussein, N.O., Sawalha, L., Khatatbeh, K. and Aldomy, A., 2009. A national survey of perinatal mortality in sheep and goats in Jordan. Pakistan Veterinary Journal, 29(3), 102-106.

Al-Qudah, K.M. Hailat, N.Q., Raouf, R.Y., Sharif, L.A., and Al-Domy, F.M. 2004. Seroprevalence of antibodies to Chlamydophila abortus shown in Awassi sheep and local goats in Jordan. Veterinary medicine - Czech, 49(12), 460-466

Berri, M., Rekiki, A., Boumedine, K.S. and Rodolaki, A., 2009. Simultaneous differential detection of Chlamydophila abortus, Chlamydophila pecorum and Coxiella burnetii from aborted ruminant's clinical samples using multiplex PCR. BMC Microbiology, 9: 130

Hartley, J.C., Kaye, S., Stevenson, S., Bennett, J., Ridgway, G., 2001. PCR detection and molecular identification of Chlamydiaceae species. Journal of Clinical Microbiology, 39 (9), 3072-3079.

Livingstone, M., Wheelhouse, N., Maley, S.W., Longbottom, D., 2009. Molecular detection of Chlamydophila abortus in post-abortion sheep at Oestrus and subsequent lambing. Veterinary Microbiology, 135, 134-141.

Longbottom, D. and Livingstone, M., 2006. Vaccination against chlamydial infections of man and animals. The Veterinary Journal, 171, 263-75.

Longbottom, D., Fairley, S., Chapman, S., Psarrou, E., Vretou, E., and Livingstone, M., 2002. Serological diagnosis of ovine enzootic abortion by enzyme-linked immunosorbent assay with a recombinant protein fragment of the polymorphic outer membrane protein POMP90 of Chlamydophila abortus. Journal of clinical microbiology, 40, 4235-4243.

Marsilio, F., B. Martino, C.E. Francesco and I. Meridiani, 2005. Diagnosis of ovine chlamydial abortion by PCR-RFLP performed on vaginal swabs. Veterinary Research Communications, 29: Suppl $199-106$.

Matthews, J. 1999. Abortion. In: Diseases of the Goat. Oxford, Blackwell Science. 167-186.

Mohamad, K.Y. and Rodolakis, A., 2010. Recent advances in the understanding of Chlamydophila pecorum infections, sixteen years after it was named as the fourth species of the Chlamydiaceae family. Veterinary Research, 41, 27.

Nietfeld, J.C., 2001. Chlamydial infections in small ruminants. Veterinary Clinics of North America: Food Animal Practice, 17:301-314.

O.I.E., 1996. Enzootic abortion in ewes. In: Manual of Standards for Diagnostic Tests and Vaccines. 3rd ed. O.I.E., Paris, France. 384388

Pospischil, A., Thoma, R., Hilbe, M., Grest, P., Zimmermann, D. and Gebbers J.O., 2002. Abortion in humans caused by Chlamydophila 
abortus (Chlamydia psittaci serovar 1). Schweizer Archiv für Tierheilkunde, 144, 463-6.

Rodolakis, A. and Souriau, A., 1989. Variations in the virulence of strains of Chlamydia psittaci for pregnant ewes. The Veterinary record, 125, $87-90$

Samadi, A., Ababneh, M. MK. Giadinis, N.D. and Lafi, S.Q., 2010. Ovine and caprine brucellosis (Brucella melitensis) in aborted animals in Jordanian sheep and goat flocks. Veterinary Medicine International. 2010; 2010: 458695.

Stone, D.M., Kumthekar, S., Chikweto, A., Thomas, D., Tiwari, K. and Sharma, R.N., 2012. Exposure to zoonotic abortifacients among sheep and goats in Grenada. International Journal of Animal and Veterinary Advances 4(2): 113-118.

Stuen, S. and Longbottom, D., 2011. Treatment and control of chlamydial and rickettsial infections in sheep and goats. Veterinary Clinics of North America: Food Animal Practice. 27(1):213-33.
Thomson, N.R., Yeats, C., Bell, K., Holden, M.T., Bentley, S.D., Livingstone, M., Cerdeño-Tárraga, A.M., Harris, B., Doggett, J., Ormond, D., Mungall, K.,Clarke, K., Feltwell, T., Hance, Z., Sanders, M., Quail, M.A., Price, C., Barrell, B.G., Parkhill, J., Longbottom, D., 2005. The Chlamydophila abortus genome sequence reveals an array of variable proteins that contribute to interspecies variation. Genome Research, 15(5):629-40.

Vladimir, V.D. and Andrey L.z., 2005. A new amplification target for PCR-RFLP detection and identification of chlamydiaceae species. Archives of Microbiology, 183,169-175.

Vretou, E., Loutrari, H., Mariani, L., Costelidou, K., Eliades, P., Conidou, G., Karamanou, S., Mangana, O., Siarkou, V., and Papadopoulos, O. 1996. Diversity among abortion strains of Chlamydia psittaci demonstrated by inclusion morphology, polypeptide profiles and monoclonal antibodies. Vet. Microbiol.51: 275-289. 